\title{
Genotype comparison of Candida albicans isolates from different clinical samples
}

\author{
Andra-Cristina Bostănaru ${ }^{1}$, Irina Roșca ${ }^{2}$, Bogdan Minea ${ }^{3}$, Valentin Năstasă ${ }^{1}$, \\ Liliana Foia $^{3}$, Iosif Marincu ${ }^{4}$, Mihai Mares ${ }^{1 *}$, Ovidiu-Alexandru Mederle ${ }^{4}$ \\ 1. Ion Ionescu de la Brad University, Iași, Romania \\ 2. Petru Poni Institute of Macromolecular Chemistry, Iasi, Romania \\ 3. Gr. T. Popa University of Medicine and Pharmacy, Iași, Romania \\ 4. Victor Babes University of Medicine and Pharmacy, Timisoara, Romania
}

\begin{abstract}
Background: Fungal infections are a health issue paradoxically fuelled by the developments in medical care. Objectives: Our study is an investigation on the correlation between the infection site and the genotypes of Candida albicans strains isolated from Romanian patients. Methods: A total number of 301 isolates from different clinical sites were investigated in terms of genotype determination. Results: The isolates were clustered in three groups according to their genotype: 55.81\% showed genotype A, $14.62 \%$ genotype B, and 29.57\% genotype C. Conclusions: No significant correlation was found between the genotype and the infection site, but a significant correlation was found between genotype C and isolates from HIV patients proving that C. albicans pathogenicity probably relies on factors related to the host.
\end{abstract}

Keywords: C. albicans, clinical source, genotype C, HIV-patients, Romania

Received: 22 $2^{\text {nd }}$ January 2019; Accepted: 11 th April 2019; Published: $18^{\text {th }}$ June 2019

\section{Introduction}

Fungal infections are a major health issue counterintuitively fuelled by the developments in medical care. The great majority of clinically relevant fungi are opportunistic pathogens for patients who receive immunosuppressive therapy, broad spectrum antibiotics, chemotherapy, patients with diabetes or HIV infection, etc. Candida yeasts are the most frequent fungal pathogen in humans, being the fourth cause of bloodstream infections [1], and of these C. albicans is the dominant species. Although much progress has been made in understanding the phenotypic and genotypic C. albicans profiles, still less is known regarding their interaction with the host [2]. This article is an extension of the previous paper dealing with Candida albicans isolates in Romanian patients (see Reference No. 3), with new results from a further elaborated analysis.

\footnotetext{
*Corresponding author: Mihai Mares, "Ion Ionescu de la Brad" University Iasi, Romania.

E-mail: mihaimares@fungi.ro
} 


\section{Methods}

The investigation was performed on 301 clinical yeast isolates collected in five tertiary hospitals from different regions of Romania during 2010-2016 (i.e. Iași, București, Cluj-Napoca, Timișoara, and Tîrgu Mureș) as follows: 62 were recovered from confirmed bloodstream infections (BSI), 66 from other deep-seated mycoses (DEEP), and 173 from superficial mycoses (SUP). DEEP mycoses were represented by the following samples: urinary tract in non-catheterized patients (U), urinary tract of HIV-infected patients (U-HIV), cerebrospinal fluid from HIV-infected patients (CSF), sputum (S), sputum from tuberculosis patients (S-TB), bronchial aspirate (BA), bronchial aspirate from tuberculosis patients (BA-TB), and laparotomy surgical drain (SD). SUP infection samples were divided into: oral samples from patients with diabetes (OS-D), oral samples from pregnant patients (OS-P), oral samples from HIV-infected patients (OS-HIV), oral samples from patients with denture (OS-D), oral samples from patients with tuberculosis (OS-TB), oral samples in patients with other predisposing conditions (OSOC), gastrointestinal tract (GT), faeces samples (F), faeces from HIV-infected patients (F-HIV), vaginal discharge (VD), vaginal discharge from pregnant women (VD-P), samples from patients with balanitis $(\mathrm{B})$, onychomycosis $(\mathrm{O})$. The isolates were collected from patients who satisfy simultaneously at least two of the following conditions: low birth weight for paediatric patients $(<1500 \mathrm{~g})$, old age for adult patients $(>65$ years), insulin-dependent diabetes mellitus, recent major surgery, broad-spectrum antibiotic therapy, central venous catheter, organ transplantation, prolonged ICU stay $(>48 \mathrm{~h})$, immunosuppression (HIV infection or other predisposing conditions), total parenteral nutrition or mechanical ventilation.

The isolates were submitted to the Laboratory of Antimicrobial Chemotherapy from the
Department of Public Health of "Ion Ionescu de la Brad" University, Iasi, Romania. All isolates were checked for purity and stored in $10 \%$ glycerol at $-70^{\circ} \mathrm{C}$. The final identification was performed using ID32C strips (bioMérieux, France). For DNA extraction, 4-5 individual colonies of $C$. albicans were picked up from Sabouraud Dextrose Agar (SDA) plates and suspended in $200 \mu \mathrm{L}$ distilled water in a sterile Eppendorf tube. DNA was obtained by lysing the yeast cells at $95^{\circ} \mathrm{C}$ for $5 \mathrm{~min}$ followed by immersing in ice and centrifugation. PCR to detect the genotypes was performed according to the method previously described [4-5] using the primers CA-NT-L (5'-ATAAGGGAAGTCGGCAAAATAGATCCGTAA-3') and CA-NT-R (5'-CCTTGGCTGTGGTTTCGCTAGATAGTAGAT-3'). All PCR amplification reactions were performed in $50 \mu \mathrm{L}$ of distilled water containing $2.0 \mu \mathrm{L}$ of each primer, $2.0 \mu \mathrm{L}$ of genomic DNA $(5 \mu \mathrm{g} / \mathrm{mL})$ and one PCR bead (Ready-to-Go PCR beads; Amersham Pharmacia Biotech, Piscataway, NJ, USA). The PCR conditions were: denaturation for $5 \mathrm{~min}$ at $93^{\circ} \mathrm{C}, 40$ cycles of $93^{\circ} \mathrm{C}$ for $30 \mathrm{~s}, 55^{\circ} \mathrm{C}$ for $45 \mathrm{~s}$, and $72^{\circ} \mathrm{C}$ for $45 \mathrm{~s}$ and a final extension at $72^{\circ} \mathrm{C}$ for $10 \mathrm{~min}$. The products were analysed using DNA microfluidic chips of Experion Automated Electrophoresis System (Bio-Rad, USA). The genotypes of $C$. albicans can be divided into 5 groups by the size of DNA amplified fragments (450 bp for group A, 840 bp for group B, 450 and $840 \mathrm{bp}$ for group C, $1080 \mathrm{bp}$ for group D and $1400 \mathrm{bp}$ for group E) [5-7]. The results were submitted to a preliminary multiple correspondence analysis (MCA) which had been assessed using XIStat-Ecology 2018.7 version. Basic descriptive statistics, chi-square test, and one-way ANOVA test were used to interpret the obtained data. For ANOVA test, $\mathrm{p}<0.05$ was considered statistically significant. 


\section{Results}

According to the site from which strains were isolated, $57.47 \%$ of them were from SUP infections, $20.60 \%$ from BSI, and $21.93 \%$ DEEP (see Table 1). C. albicans strains were divided into three different groups, based on the length of polymerase chain reaction (PCR) amplification products, namely genotype A with $55.81 \%$, B with $14.62 \%$, and C with $29.57 \%$ respectively (see Figure 1). Genotypes D and E were not detected.

Regarding SUP, most of the samples were from OS-OC (24.28\%) and VD (22.54\%), while from DEEP, the majority of the samples were from $\mathrm{S}$ (43.94\%) and BA-TB (22.72\%) (see Table 1).
In many samples $(n=168)$, genotype A was the predominant genotype, like those from VD-P (64.28\%), OS-OC (57.15\%) or F (72.72\%). Genotype C was found mostly in OS-HIV (46.15\%) (Table 1). Genotype B was mostly found in samples from $U$ and SD $(33.33 \%$ and $28.57 \%$, respectively), and also from BA-TB (26.66\%). It can also be noticed that OS-HIV had genotype C (46.15\%), instead of genotype A, which was found in oral samples from other groups of patients $(66.67 \%$ to $100 \%)$.

According to the correspondence analysis, no significant correlation was noticed between the sites of detection of isolates and their genotype $(p=0.449)$. Instead, a significant correlation

Table 1. Genotype variation in $C$. albicans isolates

\begin{tabular}{|c|c|c|c|c|}
\hline \multirow[t]{2}{*}{ Sample type } & \multirow[b]{2}{*}{$\mathbf{n}$} & \multicolumn{3}{|c|}{ Genotype } \\
\hline & & $\% \mathbf{A}$ & $\%$ B & $\% \mathrm{C}$ \\
\hline Superficial mycoses (SUP) & 173 & & & \\
\hline Oral samples diabetes (OS-D) & 6 & 100 & 0 & 0 \\
\hline Oral samples pregnant (OS-P) & 4 & 100 & 0 & 0 \\
\hline Oral samples HIV (OS-HIV) & 26 & 38.46 & 15.38 & 46.15 \\
\hline Oral samples denture (OS-D) & 3 & 66.67 & 0 & 33.33 \\
\hline Oral samples TB (OS-TB) & 1 & 0 & 0 & 100 \\
\hline Oral samples other conditions (OS-OC) & 42 & 57.15 & 14.28 & 28.57 \\
\hline Gastrointestinal tract (GT) & 3 & 66.67 & 0 & 33.33 \\
\hline Faeces $(\mathrm{F})$ & 11 & 72.72 & 9.09 & 18.19 \\
\hline Faeces HIV (F-HIV) & 6 & 50 & 16.66 & 33.33 \\
\hline Vaginal discharge (VD) & 39 & 48.72 & 25.64 & 25.64 \\
\hline Vaginal discharge in pregnancy (VD-P) & 28 & 64.28 & 7.14 & 28.57 \\
\hline Balanitis (B) & 1 & 100 & 0 & 0 \\
\hline Onycomycosis $(\mathrm{O})$ & 3 & 66.67 & 0 & 33.33 \\
\hline Blood stream infections (BSI) & 62 & & & \\
\hline Blood culture & 62 & 50 & 14.51 & 35.49 \\
\hline Deep seated mycoses (DEEP) & 66 & & & \\
\hline Urine $(\mathrm{U})$ & 3 & 33.33 & 33.33 & 33.33 \\
\hline Urine HIV (U-HIV) & 1 & 0 & 0 & 100 \\
\hline Cerebrospinal fluid HIV (CSF) & 1 & 0 & 0 & 100 \\
\hline Bronchial aspirate (BA) & 2 & 50 & 0 & 50 \\
\hline Bronchial aspirate TB (BA-TB) & 15 & 53.33 & 26.66 & 20 \\
\hline Sputum (S) & 29 & 55.17 & 24.14 & 20.69 \\
\hline Sputum TB (S-TB) & 8 & 50 & 50 & 0 \\
\hline Surgical drain (SD) & 7 & 42.85 & 28.57 & 28.75 \\
\hline
\end{tabular}




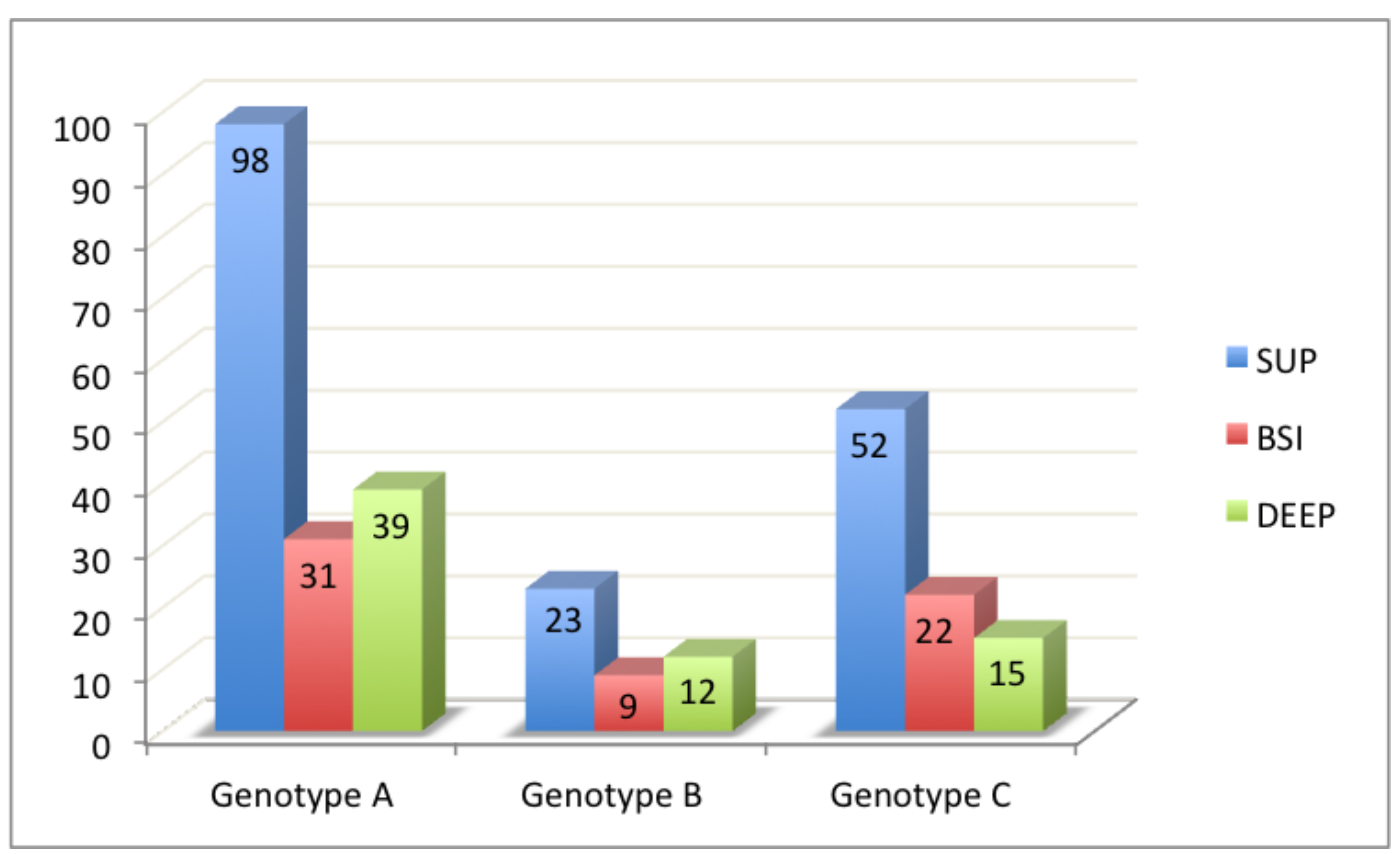

Fig. 1. Genotype distribution in C. albicans isolates from different clinical sites (absolute values: number of isolates per genotype)

between HIV-infected status and genotype C prevalence $(\mathrm{p}<0.0001)$ was observed for F-HIV, U-HIV, and OS-HIV. This fact was not found for the non-HIV samples with the same source $(\mathrm{p}=$ 0.439).

\section{Discussions}

Whether the virulence of $C$. albicans is related to genotypes remains a continuous debate. One study proves that genotype A was more prevalent among invasive strains and genotype $\mathrm{B}$ and $\mathrm{C}$ were more prevalent among none-invasive strains [8]. Al-Karaawi et al. reported that genotype $\mathrm{A}$ is the most predominant type in patients with oral Candida infections [9]. Our study proves that genotype A is not only dominant in patients with oral candidiasis, but also in patients with other SUP mycoses, DEEP, and also with BSI. Genotype determinations of $C$. albicans strains tend to be genetically similar to each other, when originating from similar pop- ulation groups, in relation to the immune condition, anatomic site, or geographical location [9]. Genotypes A, B, and C were also detected in 73 strains sampled from dental biofilms of severe early childhood caries [5]. In another study, genotypes were detected by analysing 151 strains of $C$. albicans (71 samples from infant patients with cutaneous candidiasis and 61 samples from females with vaginal candidiasis), and no distinctive association was found between genotype and the site of cutaneous infection [7]. Barros et al. found sixteen genotypes among 56 samples of $C$. albicans isolated from the oral cavity [4]. A comparison between this study and the previous ones [4-5,7] in terms of genotype is difficult due to the different number of strains and also to their prevalence in various clinical conditions, body sites or geographical conditions. Genotype A was predominant in all the examined groups and, surprisingly, genotype C was identified more frequently in isolates sampled from patients with HIV (see Table 1). Geno- 
type $\mathrm{C}$ is considered a hybrid of genotypes $\mathrm{A}$ and $\mathrm{B}$, containing the intron in only one allele [1011]. Despite a significant number of previously published studies on $\mathrm{ABC}$ genotypes in Candida albicans, no data concerning the prevalence of genotype $\mathrm{C}$ in $\mathrm{HIV}$-infected patients have been reported. Our findings could be explained by the higher resistance of genotype $\mathrm{C}$ isolates to polymorphonuclear neutrophils (PMNs) attack compared with genotypes A and B isolates [12] correlated with an impaired PMNs activity that usually occurs during HIV infection [13].

The results of our study should be interpreted in the light of some epidemiological limitations linked to an imbalanced number of isolates from different clinical sites or various general conditions of the patients.

\section{Conclusions}

No significant correlation was found between the infection site and a particular genotype. C. albicans is a diploid organism and its pathogenicity is linked to a series of inherent and environmental factors and it is mostly related to the host immunological status. However, in order to prove the above statements, our study emphasizes a significant correlation between the genotype $\mathrm{C}$ occurrence and the HIV-infected status in patients.

\section{Authors' contribution}

Andra-Cristina Bostănaru (Data curation; Formal analysis; Investigation; Writing -original draft)

Irina Roca (Data curation; Formal analysis; Funding acquisition; Investigation; Writing original draft)

Bogdan Minea (Formal analysis; Software; Writing - original draft)

Valentin Năstasă (Data curation; Formal analysis; Investigation)

Liliana Foia (Data curation; Formal analysis; Methodology)
Iosif Marincu (Conceptualization; Data curation; Investigation)

Mihai Mares, PhD (Conceptualization; Methodology; Supervision; Writing - review \& editing) Ovidiu-Alexandru Mederle (Data curation; Investigation; Methodology; Project administration; Writing - review \& editing)

\section{Declaration of conflicting interests}

The author(s) declared no potential conflicts of interest with respect to the research, authorship, and/or publication of this article.

\section{Acknowledgments}

The authors are grateful for the financial support from the H2020 ERA Chairs Project no. 667387: SupraChem Lab laboratory of Supramolecular Chemistry for Adaptative Delivery Systems ERA Chair initiative.

\section{Ethical approval}

All procedures performed in studies involving human participants were in accordance with the ethical standards of the institutional and/or national research committee and with the 1964 Helsinki declaration and its later amendments or comparable ethical standards.

\section{References}

1. Brown GD, Denning DW, Gow NAR, Levitz SM, Netea MG, White TC. Hidden Killers: Human Fungal Infections. Sci Transl Med. 2012;4(165):165rv13. DOI: 10.1126/scitranslmed.3004404

2. Odds FC, Bougnoux ME, Shaw DJ, Bain JM, Davidson AD, Diogo D, Mette DJ, et al. Molecular phylogenetics of Candida albicans. Eukaryotic cell. 2007;6(6):104152. DOI: 10.1128/EC.00041-07

3. Roșca I, Bostănaru A-C, Minea B, Năstasă V, Gherghel I, Pânzaru C-V, et al. Phenotypic and genotypic variations in Candida albicans isolates from Romanian patients. Rev Română Med Lab. 2018;26(4):405-13. DOI: $10.2478 /$ rrlm-2018-0023 
4. Barros LM, Boriollo MFG, Alves AC, Klein MI, Gonclaves RB, Hofling JF. Genetic diversity and exoenzyme activities of Candida albicans and Candida dubliniensis isolated from the oral cavity of Brazilian periodontal patients. Arch Oral Biol. 2008 Dec;53(12):1172-8. DOI: 10.1016/j.archoralbio.2008.06.003

5. Li W, Yu D, Gao S, Liu J, Chen Z, Zhao W. Role of Candida albicans secreted aspartyl proteinases (Saps) in severe early childhood caries. Int J Mol Sci. 2014 Jun 13;15(6):10766-79. DOI: 10.3390/ijms150610766

6. Tamura M, Watanabe K, Mikami J, Yazawa K, Nishimura K. Molecular characterization of new clinical isolates of Candida albicans and C. dubliniensis in Japan: analysis reveals a new genotype of C. albicans with group I intron. J Clin Microbiol. 2001 Dec;39(12):4309-15. DOI: 10.1128/JCM.39.12.4309-4315.2001

7. She XD, Wang XJ, Fu MH, Shen YN, Liu WD. Genotype comparisons of strains of Candida albicans from patients with cutaneous candidiasis and vaginal candidiasis. Chin Med J (Engl). 2008 Aug 5;121(15):1450-5. DOI: 10.1097/00029330-200808010-00021

8. Schaller M, Bein M, Korting HC, Baur S, Hamm G, Monod M, et al. The secreted aspartyl proteinases Sap1 and Sap2 cause tissue damage in an in vitro model of vaginal candidiasis based on reconstituted human vaginal epithelium. Infect Immun. 2003 Jun;71(6):3227-34. DOI: 10.1128/IAI.71.6.3227-3234.2003

9. Al-Karaawi ZM, Manfredi M, Waugh ACW, Mc-
Cullough MJ, Jorge J, Scully C, et al. Characterization of Candida spp. isolated from the oral cavities of patients from diverse clinical settings. Oral Microbiol Immunol. 2002 Feb;17(1):44-9. DOI: 10.1046/j.09020055.2001.00081.x

10. McCullough MJ, Clemons KV, Stevens DA. Molecular and phenotypic characterization of genotypic Candida albicans subgroups and comparison with Candida dubliniensis and Candida stellatoidea. J Clin Microbiol. 1999;37(2):417-21.

11. Abdulrahim MH, McManus, BA, Fliut SR, Coleman DC. 2016. Genotyping Candida albicans from Candida leukoplakia and non-Candida leukoplakia shows no enrichment of multilocus sequence typing clades but enrichment of $\mathrm{ABC}$ genotype $\mathrm{C}$ in Candida leukoplakia. PLoS One. 2013;8(9):e73738. DOI: 10.1371/journal. pone. 0073738

12. da Silva-Rocha WP, Lemos VL, Svidizisnki TI, Milan EP, Chaves GM. Candida species distribution, genotyping and virulence factors of Candida albicans isolated from the oral cavity of kidney transplant recipients of two geographic regions of Brazil. BMC Oral Health. 2014;14:20. DOI: 10.1186/1472-6831-14-20

13. Yaseen MM, Abuharfeil NM, Yaseen MM, Shabsoug BM. The role of polymorphonuclear neutrophils during HIV-1 infection. Arch Virol. 2018;163(1):1-21. DOI: 10.1007/s00705-017-3569-9 